\title{
POTENCIAL DE HÍBRIDOS DE Populusxeuramericana NA OBTENÇÃO DE POLPA CELULÓSICA
}

\author{
Silvana Mariani A. ${ }^{1}$ \\ Marco Torres U. ${ }^{2}$
}

\begin{abstract}
RESUMO
Três clones de Populusxeuramericana ( $c v$.I-72, $c v$.I-161 e $c v$. I-488) cultivados no sul do Chile foram submetidos à análise química e posteriormente a polpações kraft e soda-antraquinona. Estudou-se ainda a influência da umidade dos cavacos sobre a polpação kraft. A composição química entre os três híbridos não apresenta diferenças significativas e a polpação kraft promove o maior rendimento, menor percentagem de rejeitos e menor teor de lignina residual na polpa. O efeito do teor de umidade dos cavacos na polpação kraft exerce influência sobre o rendimento depurado, rejeito e teor de lignina residual na polpa. Um aumento na umidade dos cavacos causa um maior rendimento, menor percentagem de rejeito e menor número Kappa na polpa. Os híbridos estudados possuem um potencial favorável para a obtenção de polpa kraft, segundo os rendimentos e número Kappa similares, obtidos em espécies de Pinus spp e Eucalyptus spp.

Palavras chave: clones, propriedades químicas, kraft, soda-antraquinona, umidade
\end{abstract}

\section{POTENTIALITY OF HYBRIDS OF Populusxeuramericana FOR CELULOSE PULPING}

\begin{abstract}
Three cultivated clones of Populusxeuramericana ( $c v . I-72, c v . I-161$ and $c v . I-488)$ in southern Chile were submitted to chemical analysis and to kraft and soda-antraquinone pulping. It was still studied the influence of the chip moisture on the kraft pulping. There were no significant differences on the wood chemical composition among the three hybrids. Kraft pulping promoted the highest yield on the pulp, the lowest percentage of rejects and the lowest rate of residual lignin on the pulp. The effect of the chip moisture on the kraft pulp influences on the screened yield, percentage of rejects and the rate of residual lignin. An increase on the moisture content of chips reaches a higer yield, lower percentage of reject and lower Kappa number. According to similar yield of pulp and Kappa number reached on Pinus spp and Eucalyptus spp species pulping, the hybrids studied at these experiments own a favorable potential for obtaining kraft pulp.

Key-words: clones, chemical properties, kraft, soda-anthraquinone, moisture
\end{abstract}

\section{INTRODUÇÃO}

O cultivo do álamo (Populus sp.) no Chile ocorre desde a IV até a XI Região, com incrementos que oscilam entre 30 e 40 $\mathrm{m}^{3} /$ ha/ano e uma rotação de 12 a 16 anos, alcançando produções de 330 a $340 \mathrm{~m}^{3} / \mathrm{ha}$ (Anônimo, 1998). O álamo apresenta ainda a característica de ser uma espécie de rápido crescimento que se adapta muito bem aos solos frágeis e à margem dos rios, terrenos não aptos para outras espécies.

Devido ao potencial do cultivo de álamo e à demanda crescente pela madeira se criou no Chile uma ampla base genética, incorporando novos e melhores híbridos desde o ponto de vista silvicultural e madeireiro, gerando exemplares que alcançam densidades básicas de $348 \mathrm{~kg} / \mathrm{m}^{3}$, (Hernández et al., 1998).

Buscando novas opções produtivas para estas madeiras melhoradas, alguns pesquisadores estudaram o potencial de polpação de híbridos de álamo (Goyal et al., 1999), obtendo bons níveis de deslignificação com altos rendimentos e menor tempo de refinação das fibras, comparando-se com algumas espécies comerciais utilizadas na área celulósica, (Zarges et al., 1980).

\footnotetext{
${ }^{1}$ smariani@uach.cl. Engenheiro Civil-Químico, M. Cs., Professora da Facultade de Ciências Florestais, Universidad Austral de Chile 2 mtorres2@uach.cl. Técnico em Celulose e Papel, Professor da Facultade de Ciências Florestais, Universidad Austral de Chile
} 
Os clones de Populusxeuramericana cv.I-72, cv.I-161 e cv.I-488, cultivados em Valdivia, Chile, foram melhorados para aumentar sua produtividade em serrarias. Apesar de estar comprovado que o manejo genético de poucos caracteres pode levar a uma seleção negativa dos caracteres não controlados, a seleção do híbrido Populusxeuroamericana para melhorar seu uso em serrarias promoveria igualmente uma melhora nas qualidades químicas da madeira e na polpação. Espera-se com este estudo avaliar as características químicas e físicas da madeira e da polpa celulósica dos clones para observar o efeito do melhoramento genético sobre a espécie e suas propriedades químicas e de polpação e visualizar o seu potencial de uso na indústria de celulose.

\section{MATERIAIS E MÉTODOS}

A matéria-prima corresponde a três clones de Populusxeuramericana (P. deltoides x P. Nigra), dos cultiuvares cv.I-72, cv.I-161 e cv.I-488, os quais foram obtidos por propagação vegetativa na região de Valdivia, X Região do Chile (39³8' S), a uma altitude aproximada de 3 m.s.n.m.. O solo é do tipo "trumao", com alta porosidade e elevada capacidade de retenção hídrica. O clima é temperado úmido, com temperatura média anual entre $10^{\circ} \mathrm{C}$ e $12^{\circ} \mathrm{C}$ e precipitação média anual acima dos $2.000 \mathrm{~mm}$ (Subiabre e Rojas, 1994).
A partir de mostras de toras de cada clone, foram obtidos cavacos em forma manual para eliminar a variação dimensional nos ensaios. Os cavacos foram acondicionados e homogeneizados através de secagem ao ar livre para alcançar uma umidade de equilíbrio média de $10 \%$ base úmida.

Umidade base úmida (b.u.) $=$ (Peso úmido Peso seco)*100 / Peso úmido

Uma parte dos cavacos foram destinados à caracterização química quantitativa para cada cultivar através das normas TAPPI. Se determinaram o teor de extraíveis solúveis em água fria e solúveis em água quente pela norma T 212 om 88; solúveis em etanol-tolueno e solúveis em etanol pela norma T 204 om 88; teor de cinzas pela norma $\mathrm{T} 15$ om 88 ; teor de lignina pela norma T 22 om 88; teor de holocelulose pelo método Poljak (Navarro, 1970); e densidade básica pela norma $\mathrm{T} 258$ om 85 .

A outra fração de cavacos foi empregada na polpação Kraft e Sodaantraquinona em quente. As polpações foram realizadas em digestor $\mathrm{M} / \mathrm{K}$ System com recirculação de licor sob as condições detalhadas na tabela 1.

Posteriormente a estas polpações, avaliaram-se as polpas através dos valores de rendimentos depurado, total e de rejeitos, teor de lignina residual na polpa, através do número Kappa e análise do licor negro. 
Tabela 1: Condições nas polpações Kraft e soda-antraquinona

Table 1: Pulping conditions kraft and soda-anthraquinone

\begin{tabular}{|c|c|c|}
\hline Parâmetros polpações Kraft & Condição 1 & Condiçao 2 \\
\hline Álcali ativo $\left(\%\right.$ bms $\left.\mathrm{Na}_{2} \mathrm{O}\right)$ & 16 & 16 \\
\hline Sulfidez $(\%)$ & 22 & 22 \\
\hline Relação licor/madeira (1/kg) & $5 / 1$ & $5 / 1$ \\
\hline Temperatura máx. $\left({ }^{\circ} \mathrm{C}\right)$ & 162 & 170 \\
\hline Tempo até $\mathrm{T}^{\mathrm{o}}$ máxima (minutos) & 35 & 50 \\
\hline Tempo à $\mathrm{T}^{\mathrm{o}}$ máxima (minutos) & 90 & 60 \\
\hline Fator $\mathrm{H}$ & 734 & 1048 \\
\hline Parâmetros polpações soda-antraquinona & \multicolumn{2}{|c|}{ Condição única } \\
\hline Soda $(\%$ bms $)$ & \multicolumn{2}{|c|}{20} \\
\hline Antraquinona (\%bms) & \multicolumn{2}{|c|}{0,15} \\
\hline Relação licor/madeira (1/kg) & \multicolumn{2}{|c|}{$5 / 1$} \\
\hline Temperatura máx. $\left({ }^{\circ} \mathrm{C}\right)$ & \multicolumn{2}{|c|}{162} \\
\hline Tempo até Tmáx. (minutos) & \multicolumn{2}{|c|}{50} \\
\hline Tempo à T máx. (minutos) & \multicolumn{2}{|c|}{120} \\
\hline
\end{tabular}

*bms: base madeira seca.

Finalmente, aplicou-se uma polpação kraft aumentando o factor $\mathrm{H}$, a qual denominou-se condição 2 como se observa na tabela 1. Com a condição 2 de polpação aplicou-se duas condições de umidade nos

Tabela 2:Umidade dos cavacos dos híbridos

Table 2: Chips moisture content hybrids

\begin{tabular}{lcc} 
& \multicolumn{2}{c}{ Umidade cavacos (\% base úmida) } \\
\hline Clone & Secos ar & Saturados \\
\hline$c v . I-72$ & 10,5 & 71,4 \\
$c v . I-161$ & 11,9 & 76,1 \\
$c v . I-488$ & 9,7 & 73,2 \\
\hline
\end{tabular}

\section{RESULTADOS}

Análises químicas e físicas da madeira dos clones: na Tabela 3 se apreciam os valores das determinações químicas para cada clone de Populusxeuramericana.

Os valores para os extraíveis solúveis em água fria e quente, $\mathrm{NaOH} 1 \%$, etanoltolueno e extraíveis totais na madeira dos clones cv.I-72 e cv.I-161 são praticamente da mesma ordem, sendo que o clone cv.I-488 apresenta menor quantidade de extraíveis, por exemplo $10 \%$ menos extraíveis totais. Esta propriedade deveria repercutir em uma maior cavacos para cada clone conforme se observa na tabela 2. Neste caso, para alcançar o maior teor de umidade nos cavacos foi necessário aplicar um banho em água fria durante 48 horas.

deslignificação pois haverá menor competição dos extraíveis pelos reativos destinados a este processo. O teor de cinzas nos três clones está dentro da média de ocorrência observada na bibliografia para tais valores (Fengel, 1989) flutuando entre 0,5 e $0,8 \%$ bms (base madeira seca) de material inorgânico e maior para os clones cv. I-161, enquanto que o teor de holocelulose não apresenta diferenças significativas entre os clones. Com respeito ao teor de lignina, a diferença entre os clones não supera a $10 \%$ por que seu efeito sobre a polpa seria mínimo. 
Tabela 3: Análise química quantitativa dos clones

Table3: $\quad$ Cuantitative chemical analysis of clones

\begin{tabular}{lccc}
\hline \multicolumn{1}{c}{$\begin{array}{c}\text { Determinações } \\
\text { Químicas }\end{array}$} & \multicolumn{3}{c}{ Populusxeuramericana } \\
\hline \multicolumn{1}{c}{$\%$ bms* } & $c v . I-72$ & $c v . I-161$ & $c v . I-488$ \\
\hline Solúveis em: & 4,2 & 4,6 & 3,8 \\
Agua fria & 3,9 & 4,1 & 3,7 \\
Agua quente & 20,8 & 20,8 & 21,3 \\
NaOH a 1\% & 3,1 & 3,1 & 2,9 \\
Etanol-tolueno & 6,4 & 6,4 & 5,8 \\
Extraíveis totais & 0,6 & 0,8 & 0,5 \\
Cinzas & 22,8 & 24,9 & 23,8 \\
Lignina (bmle)** & 68,0 & 68,3 & 67,9 \\
Holocelulose (bmle) & & & \\
\hline
\end{tabular}

*bms: base madeira seca.

** bmle: base madeira livre de extraíveis.

Os valores das determinações de densidade dos três clones são apresentadas na figura 1 .

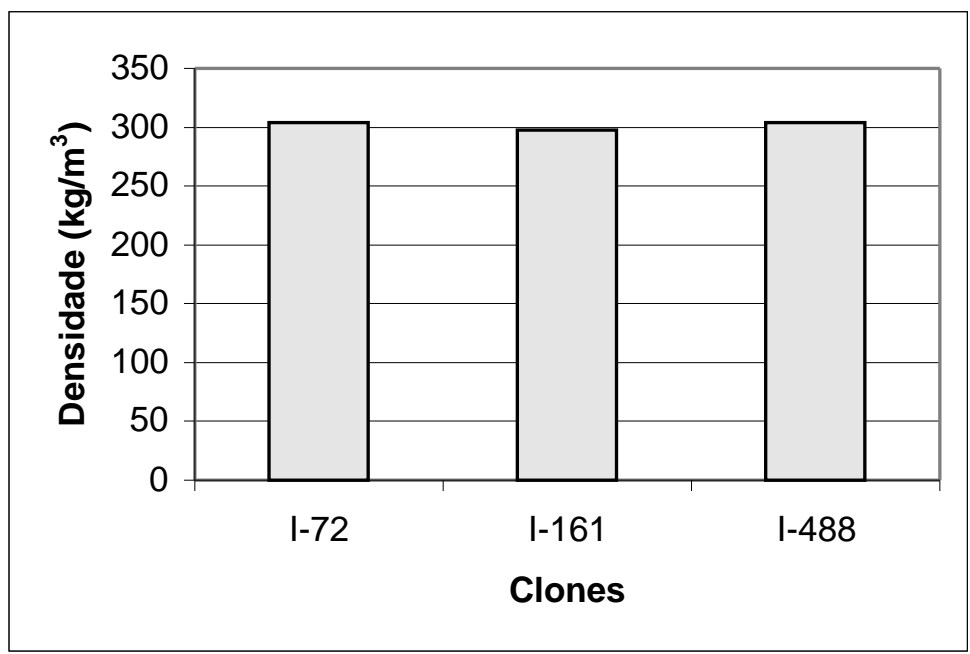

Figura 1: Densidade básica dos três clones de Populusxeuramericana.

Figure 1: Basic density of the wood of the clones Populusxeuramericana

Se nota claramente que os clones cv. I72 e cv.I-488 apresentam uma densidade semelhante, enquanto que os clones cv. I-161 apresenta uma densidade levemente mais baixa comparada com os demais (2,1\% menor), porém esta diferença é insignificante considerando que o erro admitido pela norma para este ensaio é menor à diferença encontrada.

Clones nos processos de polpação Kraft e soda quente com antraquinona: nas figuras 2 e 3 se observa os rendimentos e número de Kappa na polpa para ambos processos. 


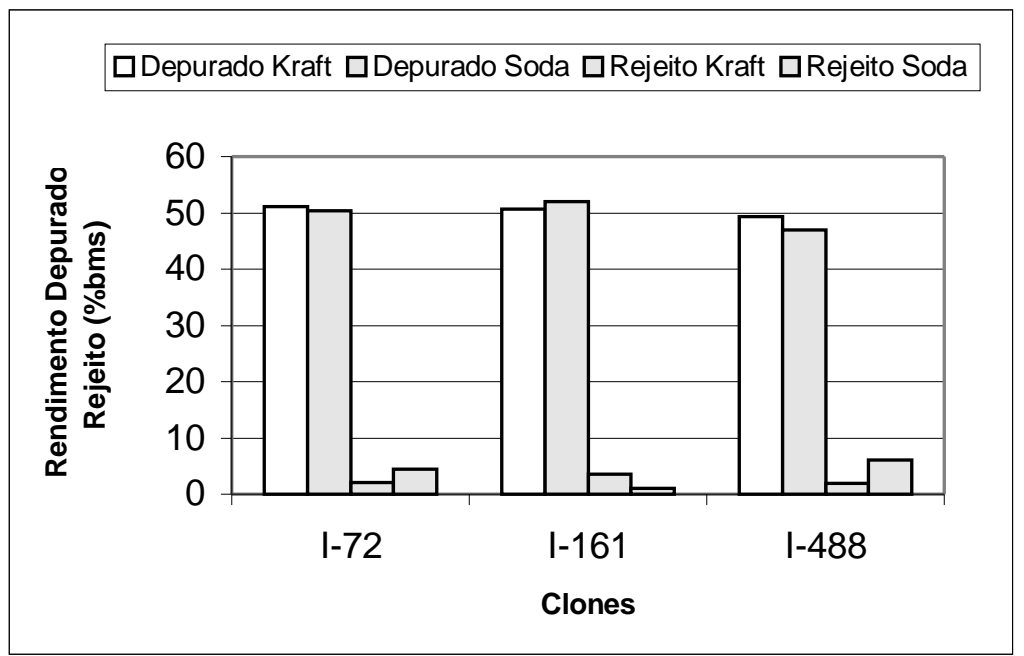

Figura 2: Rendimentos nas polpações

Figure 2: Celulose cooking yield

O maior rendimento depurado e os menores rejeitos para os clones cv.I-72 e cv.I488, foram obtidos com o processo Kraft, enquanto que para o clone cv.I-161 este se alcança durante a polpação soda. Nos processo soda-antraquinona os clone cv.I-72 e cv.I-488 apresentam os maiores rendimentos totais, devido à elevada percentagem de rejeitos obtida, como se aprecia na figura 2 .
No que se refere aos números de Kappa na polpa (fig. 3) observa-se claramente a vantagem que apresenta o processo Kraft. Os clones $c v$. I-72 e $c v$.I-488 renderam polpas no processo Kraft com número de Kappa $50 \%$ menor, comparadas ao processo sodaantraquinona.

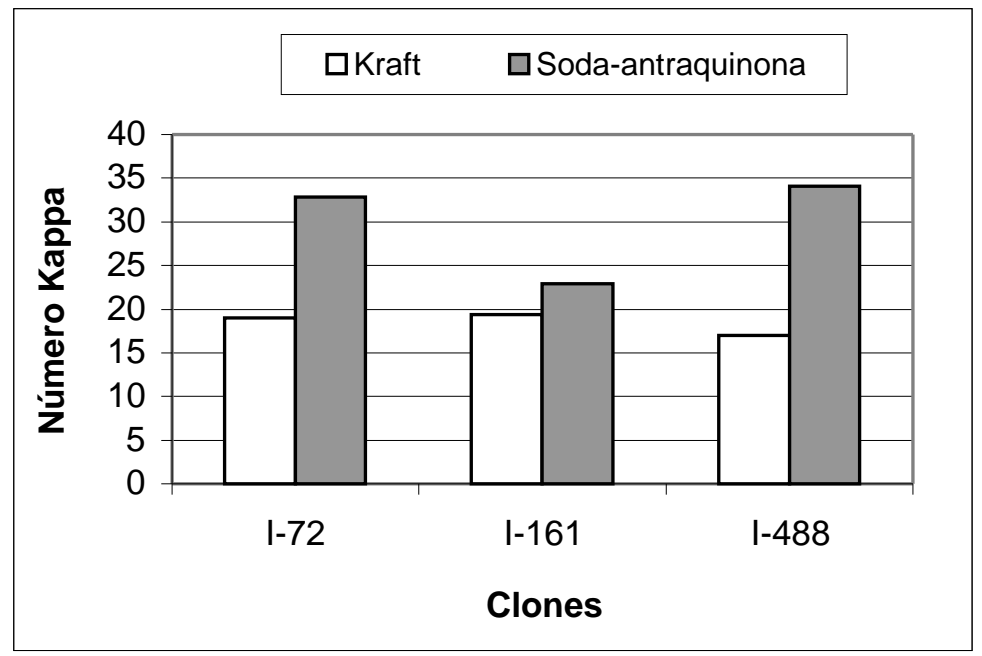

Figura 3: Numero de Kappa das polpas

Figure 3: Kappa number on the pulp

Efeito da variação da umidade nos cavacos de clones na polpação Kraft: as condições de polpação utilizadas para esta análise é a condição 2 apresentada na Tabela 1. Observando a tabela 4 , nota que uma diferença de cerca de $60 \%$ na umidade dos cavacos, provoca um aumento do rendimento depurado entre 2,0 e $8,0 \%$, dependendo do clone tratado. Os maiores efeitos da umidade se observam na percentagem de rejeitos e na desliginificação da polpa. O incremento da umidade dos cavacos reduz entre 87 a $93 \%$ a quantidade de rejeitos e entre 17 e $22 \%$ o teor de lignina residual na polpa, ou seja, o aumento da 
umidade nos cavacos proporcionou uma maior possibilidade de penetração dos reativos de polpação até o centro do cavaco e através das paredes celulares para reacionar com a lignina.

Tabela 4: Respostas na polpa kraft obtida com níveis de teor de umidade dos cavacos

Table 4: Answers in kraft pulp obtained with chips in two levels of humidity

\begin{tabular}{|c|c|c|c|c|c|c|}
\hline \multirow[t]{2}{*}{ Clones } & \multicolumn{2}{|c|}{ Rendimento depurado (\%bms) } & \multicolumn{2}{|c|}{ Rejeito (\%bms) } & \multicolumn{2}{|c|}{ Número Kappa } \\
\hline & Secos ao ar & Saturados & Secos ao ar & Saturados & Secos ao ar & Saturados \\
\hline$c v \cdot \mathrm{I}-72$ & 49,4 & 51,3 & 3,6 & 0,3 & 16,7 & 13,9 \\
\hline$c v \cdot \mathrm{I}-161$ & 51,1 & 52,3 & 2,4 & 0,3 & 18,0 & 13,9 \\
\hline$c v \cdot \mathrm{I}-488$ & 49,3 & 53,0 & 2,9 & 0,2 & 22,9 & 19,0 \\
\hline
\end{tabular}

Também pode-se apreciar que independente da condição de polpação Kraft (diferenças de umidade nos cavacos ou fator $\mathrm{H})$ os três clones apresentam uma favorável capacidade de polpação no que se refere às porcentagens de rendimento classificado e rejeitos, porém quando avaliasse os Números de Kappa para as duas condições de umidade, o clone cv.I-488 apresenta maior dificuldade para deslignificação, obtendo-se uma polpa com número de Kappa 35\% superior às polpas dos demais clones. Se estes resultados são analisados conjuntamente com as baixas diferenças quantitativas nos componentes químicos e de densidade da madeira entre os clones, podemos inferir que a diferença na reatividade da lignina depende do tipo de lignina ou das porcentagens de cada tipo de lignina presentes nos clones $c v . \mathrm{I}-72, c v . \mathrm{I}-161$ comparados com $c v$.I-488.

$\mathrm{Na}$ tabela 5 observam-se os rendimentos volumétricos alcançados com o processo Kraft e o nível de deslignificação para cada polpa. Os três clones apresentam altos rendimentos volumétricos proporcionando polpas de grau branqueável sob as condições de cozimento aplicadas.

Tabela 5: Índices comparativos das polpações dos clones de Populusxeuramericana*.

Table 5: Comparative pulping indexes hybrids Populusxeuramericana*.

\begin{tabular}{lcccc}
\hline Clones & $\begin{array}{l}\text { Densidade } \\
\left(\mathrm{kg} / \mathrm{m}^{3}\right)\end{array}$ & $\begin{array}{c}\text { Rendimento } \\
\text { Volumétrico } \\
\left(\mathrm{kg} / \mathrm{m}^{3}\right)\end{array}$ & $\begin{array}{l}\text { Número } \\
\text { Kappa }\end{array}$ & $\begin{array}{l}\text { Sólidos } \\
\text { Licor \% } / \mathrm{p}\end{array}$ \\
\hline$c v . \mathrm{I}-72$ & 304,0 & 156 & 13,9 & 14,5 \\
$c v . \mathrm{I}-161$ & 297,8 & 156 & 13,9 & 14,1 \\
$c v . \mathrm{I}-488$ & 304,1 & 161 & 19,0 & 12,9 \\
\hline
\end{tabular}

* Valores das polpações realizadas com cavacos saturados acima de $70 \%$ b.u.

\section{CONCLUSÕES}

- A composição química quantitativa dos três clones Populusxeuramericana cv.I-72, $c v$. I-161 e $c v$. I-488 é praticamente similar, exceto no teor de lignina, onde a diferença máxima não supera a $10 \%$.

- Não se apresenta diferença quanto aos valores de densidade da madeira nos três clones.

- O processo de polpação Kraft proporciona maiores rendimentos, menores percentagens de rejeito e diminuição de $50 \%$ em número de Kappa comparados com o processo sodaantraquinona, apresentando valores mais atrativos para o clone $c v$.I-161.

- No processo Kraft, o efeito da umidade nos cavacos parece ser uma variável importante já que esta aumenta o rendimento depurado junto com a diminuição de rejeitos e teor de lignina residual na polpa.

- Independente das condições de cozimento, os clones $c v$.I-161 e $c v$. I-72 apresentam um maior grau de deslignificação (menor número de Kappa) comparados com o clone $c v . I-488$. Devido às primeiras conclusões que afirmam maiores diferenças na reatividade da lignina comparadas às baixas diferenças na quantidade, permitem presumir que existem variações no tipo de lignina entre os clones.

\section{REFERÊNCIAS}

ANÔNIMO,1998."Alternativa para el agro", Chile Forestal No261:36-38.

GOYAL G., FISHER J., KROHN M., PACKOOD R., OLSON J., 1999, "Variability in pulping and fiber characteristics of hybrid 
poplar tress due to their genetic makeup, enviromental factors, and tree age", Tappi Journal, 82(5):141-147.

FENGEL D., WEGENER G.1989. Chemistry, Ultastructure, Reactions. Walter de Gruyter. New York. 613 pp.

HERNÁNDEZ R., KOUBAA A., BEAUDOIN M., FORTIN E. 1998. "Selected mechanical properties of fast-growing poplar hybrid clones", Wood and Fiber Science, 30 (2):138-147.
NAVARRO J.1970. Temas de la fabricación del Papel. Ed. Marfil S.A. España. 97 pp.

SUBIABRE A., ROJAS C. 1994. Geografía física de la Región de Los Lagos. Ed Universidad Austral de Chile, Valdivia, Chile, 117 .

ZARGES R., NEUMAN R., CRIST J., 1980, "Kraft pulp and paper properties of Populus clones grown under short-rotation intensive culture", Tappi, 63(7):91-94. 